\title{
Investigating the use of Boyle's Law to relate pore air pressures and volume changes in unsaturated triaxial samples
}

\author{
Katherine $\mathrm{Kwa}^{1, *}$ and David Airey ${ }^{1}$ \\ ${ }^{1}$ The University of Sydney, School of Civil Engineering
}

\begin{abstract}
Measuring the volume changes of unsaturated triaxial soil samples is one of the main challenges when performing unsaturated triaxial tests. Under fully undrained conditions, Boyle's Law can be used to calculate the sample's volume changes caused by the compression of air, as Boyle's Law relates changes in volume to changes in pore air pressures within the sample. This method has been used to calculate the volumetric strains and pore air pressures in unsaturated samples tested under cyclic loading conditions. However, the volume changes calculated from using Boyle's Law and the increases in pore air pressures, have not been compared with the actual measured volume changes of unsaturated samples. This study presents the pore pressures and compares the measured and predicted volumetric strains calculated from using Boyle's Law, in unsaturated triaxial samples that were tested cyclically. In some cases, using Boyle's Law was found to be similar to the volume changes estimated from direct measurement, but sometimes the method did not appear to work. Reasons for the discrepancies will also be discussed.
\end{abstract}

\section{Introduction}

Unsaturated soils contain 3 phases; solid soil, air and water. Therefore, according to Bishop's effective stress equation [1], the pore air and water pressures and the degree of the saturation are required to determine the effective stress within unsaturated soils. However, it can be difficult to accurately measure the pore air pressures when the air phase is discontinuous within samples with higher degrees of saturation. As an alternative to directly measuring the air pressure in unsaturated triaxial soil samples, Boyle's Law has been used to calculate the air pressures from the measured volume changes in samples of silty sand tested cyclically under unsaturated and undrained conditions in triaxial tests [2-5]. Boyle's Law states that the pressure of an ideal gas is inversely proportional to its volume at a constant temperature and it can be expressed according to Equation 1.

$$
V_{a}=\frac{\bar{u}_{a 0}}{\bar{u}_{a}} V_{a 0}
$$

where $V_{a}$ is the volume of air, $\bar{u}_{a 0}$ is the initial absolute air pressure (ie. $\bar{u}_{a 0}=u_{a 0}+\bar{u}_{a t m}$ ), $u_{a 0}$ is the initial gauge air pressure, $\bar{u}_{a t m}$ is the atmospheric pressure $(101.3 \mathrm{kPa})$, $V_{a 0}$ is the initial volume of air and $\overline{u_{a}}$ is absolute air pressure (ie. $\bar{u}_{a}=u_{a}+\bar{u}_{a t m}$ ). Boyle's Law can therefore be used to find the volumetric strains $(\epsilon)$ in unsaturated samples that are tested under undrained conditions as the volume changes in an undrained test are a result of the compression of the air present in the samples. Therefore, Equation 2 can be used to back calculate volume changes within the samples.

$$
\frac{\Delta V}{V}=-\frac{V_{a 0}}{V_{a}}\left(\frac{\bar{u}_{a t m}}{\bar{u}_{a}}-1\right)
$$

\footnotetext{
*e-mail: katherine.kwa@sydney.edu.au
}

However, the volume changes predicted from using Boyle's Law have not been validated against the actual volume changes of samples in the literature.

There are several methods that have been commonly used in unsaturated soil studies to measure the actual volume changes of samples in triaxial tests. Internal instrumentation has been used to measure the local radial and axial strains in samples [6]. Image processing methods have also been used to track the changes in volume from photographs taken of the sample [7, 8]. Alternatively, the volume changes in the cell water surrounding the sample have also been used to measure the volume change of the sample $[2,3,5,9,10]$. Monitoring the volume changes of the cell water is the more common approach, as irregularities in the sample's shape from the formation of shear planes and bulging, can make it difficult for internal instrumentation to accurately measure the global volume changes of the sample. Multiple cameras are also required to capture asymmetric deformations in the sample and there are difficulties during the calibration process to allow for the shape of the cell and the different refraction indices. Studies that use the volume changes of the water in the cell to monitor the volume changes of the sample typically use a smaller inner cell to reduce the errors in measuring the cell water volume changes, mainly caused by the cell volume changing with cell pressure, water absorption and thermal expansion as well as creep of the cell under a constant cell pressure.

This study is part of a larger project that explores the unsaturated cyclic performance of bulk metallic ore cargoes that are known to liquefy when subjected to wave rocking motions during maritime transportation. These ore cargoes are transported moist and at relatively low 
densities. They are well graded materials, containing a wide range of particle sizes $(9.5 \mathrm{~mm}$ to less than $0.075 \mathrm{~mm}$ in diameter), which are not commonly tested in earthquake induced liquefaction studies. In this study, unsaturated samples of an artificial material, similar in grading to the metallic ore cargoes have been tested under cyclic undrained conditions at degrees of saturation between 55 and $90 \%$ in a modified triaxial apparatus. In particular, the effects of the degree of saturation, cyclic stress ratio (CSR) and density on the pore air and water pressure responses and the volumetric response of samples, have been investigated. In particular, the pore air pressures measured from testing the artificial materials will also be used to verify Boyle's Law through comparing the theoretical volume changes calculated from the air pressures within the sample, with the measured volume changes of the samples from changes in the cell volume.

\section{Methodology}

Samples were reconstituted and contained a mixture of angular basalt aggregates with moderate sphericity, ranging in particle size from $9.5 \mathrm{~mm}$ down to $0.015 \mathrm{~mm}$, and sub-rounded, non-plastic feldspar (60\% orthoclase and 10$30 \%$ albite) fines of moderate sphericity. Samples contained $18 \%$ fines, where fines are defined as particles less than $0.075 \mathrm{~mm}$ in size. Samples were prepared for triaxial testing by compacting the moist soil in five layers in a $200 \mathrm{~mm}$ high $\times 100 \mathrm{~mm}$ diameter split mould, to relative densities similar to those that occur from the loading process of the bulk metallic ore cargoes into the hold of a ship [11]. The compacted samples were then carefully mounted in a triaxial apparatus and brought to their initial target degree of saturation (between 50 and $90 \%$ ) by applying a small pressure $(<5 \mathrm{kPa})$ to the base and pumping water into the sample while leaving the air line at the top of the sample open to atmosphere. Once the target saturation was reached, further drainage from the top of the sample was prevented to minimise water coming out of the sample through the air line and affecting the pore air pressure transducer readings during subsequent testing stages. The samples were then anisotropically consolidated by initially isotropically consolidating samples under a confining pressure of $200 \mathrm{kPa}$ and then by increasing the deviator stress to $\mathrm{q}=200 \mathrm{kPa}$ while maintaining a constant cell pressure. This anisotropic stress state is believed to be representative of the stresses in an element located in the middle of the base of a heap of iron ore fines when loaded into a bulk carrier ship [11]. Samples were left at this stress state until equilibrium was achieved. Once the samples had reached equilibrium, the tap that allowed drainage of water from the sample was turned off and the samples were then cyclically loaded under undrained conditions at various CSRs, either until failure (defined as axial strain exceeding 5\%), or until 3000 cycles had been applied. This was the relevant number of cycles that the cargo is subjected to in a severe storm during transportation [11]. Samples were subsequently sheared monotonically to failure, however, this study focuses mainly on the responses of the samples during the cyclic loading stage.

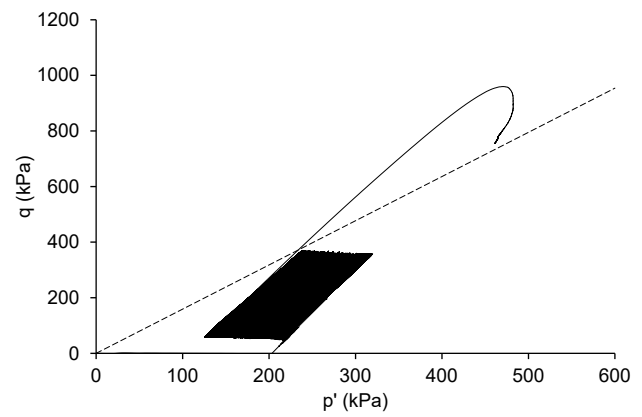

Fig. 1. Typical Stress Path of Samples in $q$ vs $p^{\prime}$ space

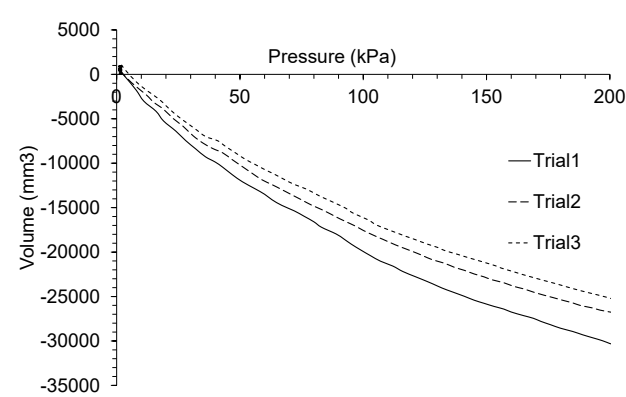

Fig. 2. Cell Water Volume Changes from Changes in Pressure

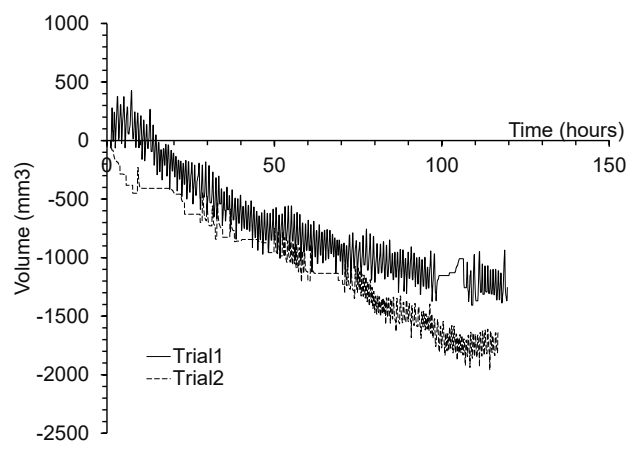

Fig. 3. Cell Water Volume Change at a Constant Pressure

A typical stress path of a test in the $q$ vs $p^{\prime}$ space is shown in Fig. 1.

The unsaturated triaxial testing methods used in this study were similar to those described in $[12,13]$. The sample's pore water pressures were measured at the base of the sample and to prevent air from diffusing through the bottom of the sample, a 3 bar high air entry porous disk was embedded into the base of the triaxial pedestal. The high air entry disk was deaired regularly between tests and the water lines and pore water pressure transducer were flushed with deaired, distilled water. To measure the air pressure changes in the sample, a pressure transducer filled with air was connected to the drainage at the top of the sample. To prevent water travelling from the sample into the top drainage line and affecting the pore air pressure readings, a very coarse porous disk was also placed 
at the top of the sample and below the top platen. The air line, pressure transducer and coarse porous disk were also checked for any traces of water and dried between tests. The volume change of the sample was measured through monitoring the volume of deaired water flowing in and out of the triaxial cell. The samples tested in this study had a diameter of $100 \mathrm{~mm}$, an approximate height of $190 \mathrm{~mm}$ and therefore a volume of $1,492,000 \mathrm{~mm}^{3}$. These samples were much larger than the unsaturated samples of silty sand tested in $[2,3,5,9,10]$ and the associated volume changes of the larger samples were significantly greater than the volume changes measured in smaller samples. Therefore, the volume changes of the unsaturated samples tested in this study were measured through monitoring the volume of water that flowed directly in and out of the triaxial cell and volume corrections that accounted for the volume measurement errors from the expansion of the cell due to creep, and due to water being absorbed into the cell wall, were applied. All volume correction errors were calculated as a percentage with respect to the total volume of an unsaturated triaxial sample.

To obtain the cell volume correction due to increases in pressure, the cell volume changes from filling the cell with deaired water and applying $200 \mathrm{kPa}$ cell pressure, the highest cell pressure used in this study, around an incompressible dummy steel sample, were determined, as shown in Fig. 2. The negative volume readings in Fig. 2 represent the amount of water flowing into the cell from a GDS volume pressure controller, as the cell pressure was increased. These recorded cell volume changes were found to be repeatable with a $0.3 \%$ variation. To find the volume changes due to creep of the cell under constant pressure, the volume changes in the cell water around the incompressible dummy steel sample were monitored for up to 5 days, the maximum time required to test the samples. These volume changes are shown in Fig. 3 and the small variations in the measured cell volume of approximately 300 to $400 \mathrm{~mm}^{3}$ (less than $0.1 \%$ of the volume of the sample) are believed to be the result of fluctuations in room temperature and were not included in the volume corrections. From Fig. 3, it is evident that the rate of the inflow of water decreased with time and the total volume change after 5 days was around 1,500 to $2,500 \mathrm{~mm}^{3}$, or approximately $0.1 \%$ of the total volume of the samples tested in this study. An extra volume correction that accounted for the volume of water that the ram $(25 \mathrm{~mm}$ in diameter) displaced was also applied and calculated from the axial displacement readings recorded by the Linear Variable Differential Transformer (LVDT). The volume changes calculated from measuring the change in volume of the cell water, and applying the volume corrections, were then validated with the volume changes measured in 6 saturated samples tested under standard, monotonic drained conditions. The standard method for measuring volume changes in saturated samples that are tested under drained conditions, is to monitor the flow of pore water in and out of the sample. The resulting sample volume changes from using the recorded volume changes in the cell water with the applied volume corrections, and from using the standard

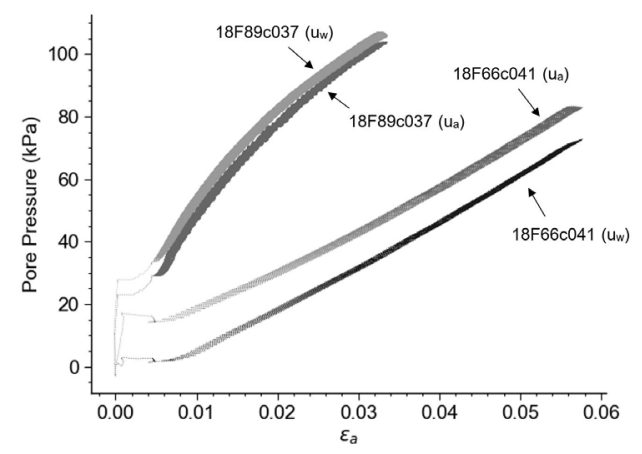

Fig. 4. Typical Pore Pressure Response

method were found to be within less than $\pm 0.1 \%$ of each other.

\section{Results and Discussion}

The samples tested in this study are named and labelled according to the degree of saturation achieved after the consolidation stage and the CSRs that were used. For example, test $18 \mathrm{~F} 89 \mathrm{c} 037$ reached a degree of saturation of $89 \%$ after the consolidation stage and was tested at a CSR of 0.37. The pore air and water pressures that were measured in the samples shown in the following figures are labelled with the name of the test followed by $\left(u_{a}\right)$ or $\left(u_{w}\right)$ for pore air and water pressure respectively. Fig. 4 shows the typical pore air and water pressure responses of the unsaturated samples, tested at 66 and $89 \%$ degree of saturation, with respect to axial strains during the consolidation and cyclic loading stages of the test. The initial jumps in the pore pressures at $0 \%$ axial strain were a result of preventing the drainage of air during the consolidation stage. Both the pore water and air pressures subsequently increased significantly during the cyclic loading stage as samples compressed and also increased in degree of saturation. The pore water and air pressure responses tended to be very similar and as a result, only small suctions developed in the samples (where suction is the difference between the pore air and water pressures). These observations are consistent with the typical pore pressure responses observed in unsaturated samples of silty sand that were tested under undrained conditions, at relatively high degrees of saturation above $60 \%$ and at low suctions in other liquefaction studies [2, 5,9].

Fig. 5 shows the typical effect of degree of saturation on the pore water and air pressure responses with respect to axial strain during the cyclic loading stage of samples prepared at approximately the same densities and tested at similar CSRs. Samples collapsed by varying amounts during the consolidation stage, which caused small variations in the samples' void ratios and degrees of saturation. There was only a small difference between the pore air and water pressures measured in the samples tested within the range of saturations that were used in this study. The general trends in Fig. 5 show that as the degree of saturation was increased, samples experienced more rapid increases in the pore pressure. Towards the end of the cyclic 


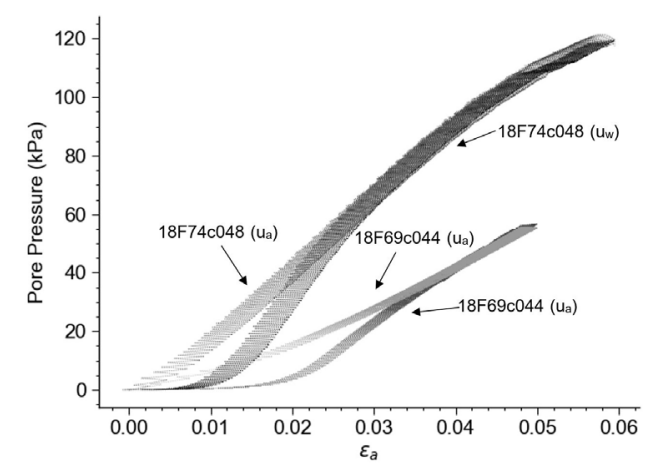

Fig. 5. Effect of Degree of Saturation on the Pore Pressures

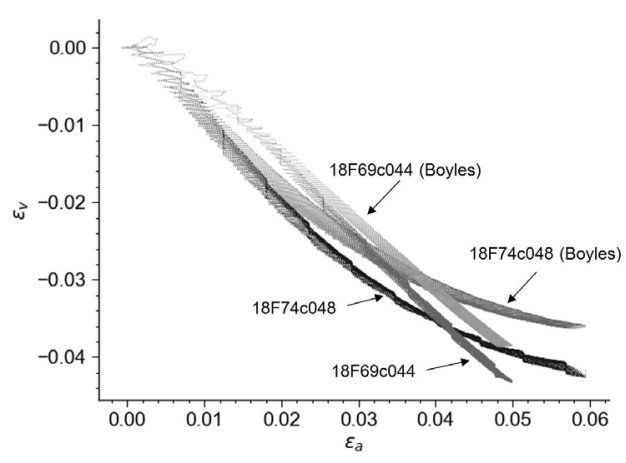

Fig. 6. Effect of Degree of Saturation on $\epsilon_{v}$

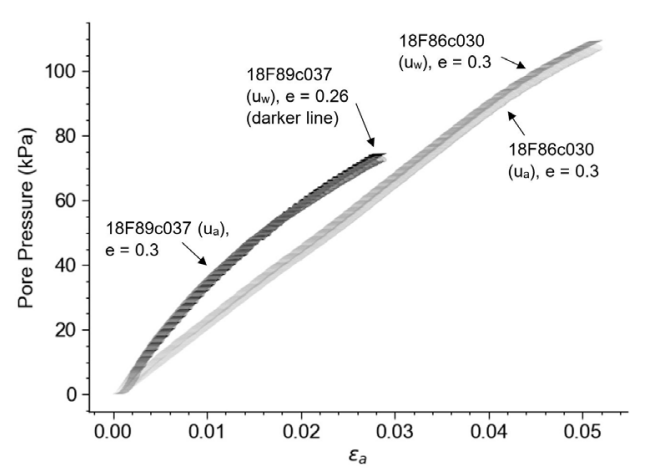

Fig. 7. Effect of Density on the Pore Pressures

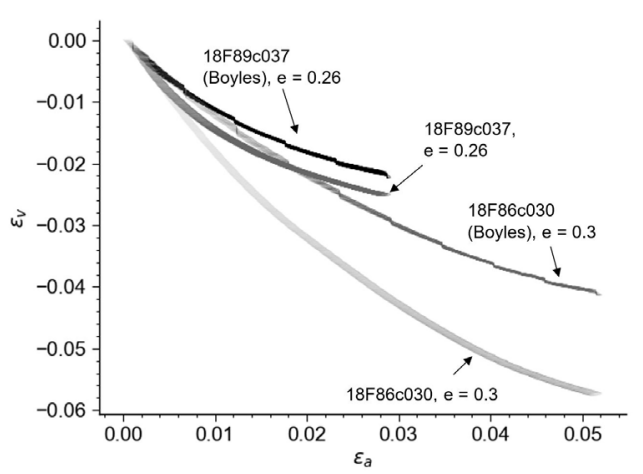

Fig. 8. Effect of Density on $\epsilon_{v}$

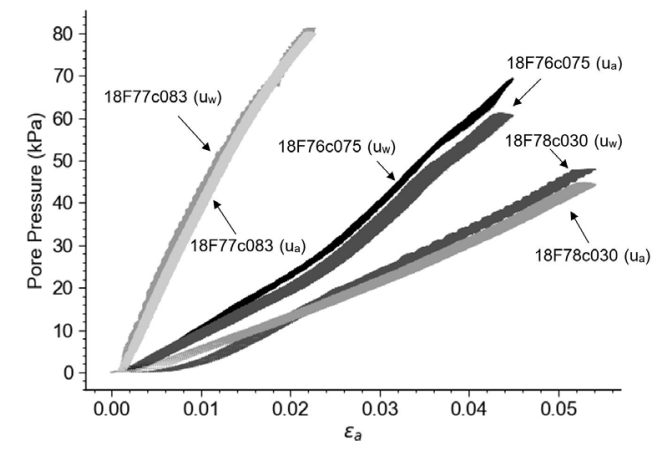

Fig. 9. Effect of CSR on the Pore Pressures

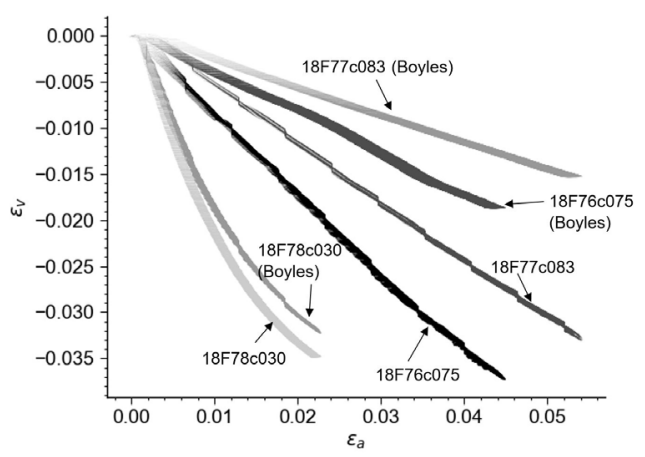

Fig. 10. Effect of CSR on $\epsilon_{v}$

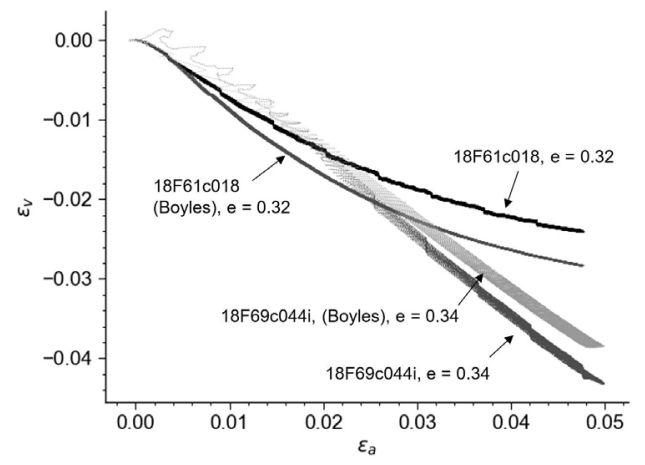

Fig. 11. Volumetric Strains Measured in Loose Samples



Fig. 12. Volumetric Strain Errors in Samples 
loading stage, the pore water pressures measured in test $18 \mathrm{~F} 74 \mathrm{c} 048$ increased to pressures similar in value to the pore air pressures at lower values of axial strain than test $18 \mathrm{~F} 74 \mathrm{c} 048$. This is because test $18 \mathrm{~F} 74 \mathrm{c} 048$ was prepared at a higher degree of saturation than test $18 \mathrm{~F} 69 \mathrm{c} 044$ and therefore, the increase in pore pressure is more likely to be reflected by increases in both the air and water phases, both which are likely to be continuous phases within the samples. However, if the degree of saturation is increased further, then the air phase starts to becomes discontinuous, present as bubbles within a continuous water phase Therefore, increase in pore pressures within samples with higher degrees of saturation are more likely to be reflected by increases in pore water pressures rather than the air pressures, as can be seen by test $18 \mathrm{~F} 86 \mathrm{c} 030$ presented in Fig. 7. This resulted in small negative suctions of $-2 \mathrm{kPa}$ during the cyclic loading stage of the test. Fig. 7 compares the typical pore air and water pressure responses in samples prepared at different densities. Test $18 \mathrm{~F} 86 \mathrm{c} 030$ was prepared slightly looser (void ratio of 0.3 ), than test $18 \mathrm{~F} 89 \mathrm{c} 037$ (void ratio of 0.26 ), experienced a greater build up in pore pressures, compressed more and cyclically failed within 3000 cycles when loaded at the same CSR as test 18F89c037. Fig. 9 shows the typical effect of CSR on the pore pressure responses in samples. As the CSR was increased, the build up in pore pressures decreased and this is because the pore pressures required to reach failure in the $q$ vs $p^{\prime}$ space also decreased. All the samples shown in Fig. 9 were tested at relatively high degree of saturations, corresponding to when the air phase begins to become discontinuous. This is believed to be why the measured pore water pressures were slightly higher than the pore air pressures in these samples.

The corresponding volumetric responses $\left(\epsilon_{v}\right)$ measured from using the volume changes in the cell water and from using Boyle's Law in the samples discussed earlier are shown in Figs. 6, 8 and 10. The volume changes of samples predicted from using Boyle's Law are labelled with the name of the test followed by (Boyles). The negative volumetric strain readings correspond to water flowing from the cell GDS volume pressure controller into the triaxial cell, which indicated that samples were compressing. Therefore from the measured sample volume change readings, it is evident that all of the samples compressed during the cyclic loading stage of the test, which is consistent with the measured increases in the pore air and water pressures as discussed earlier. Samples that contained more air tended to be more compressible as is evident in Figs. 6 and 8. Test $18 \mathrm{~F} 74 \mathrm{c} 048$ compressed more than test $18 \mathrm{~F} 69 \mathrm{c} 044$ because test $18 \mathrm{~F} 69 \mathrm{c} 044$ was prepared at a lower degree of saturation, and therefore, contained more air. Similarly, test $18 \mathrm{~F} 86 \mathrm{c} 030$ was prepared at approximately the same degree of saturation, but at a higher void ratio than test $18 \mathrm{~F} 89 \mathrm{c} 037$ and as a result, test $18 \mathrm{~F} 86 \mathrm{c} 030$ compressed more. Samples which were loaded at higher CSRs, also compressed more as can be seen in tests $18 \mathrm{~F} 76 \mathrm{c} 075$ and 18 F78c030 shown in Fig. 10. Test 18 F77c083 was prepared slightly denser than the other tests (at a void ratio of 0.24 compared to void ratios of 0.3 ), which is why it did not fit with the trend in Fig. 10.
The theoretical volume changes from using the measured pore air pressures and Boyle's Law also predicted compression in the samples, however, the predictions tended to underestimate the compressive volume changes in the samples. It may be expected that Boyle's Law would not be as effective in predicting the volume changes in samples with higher degrees of saturation where the air phase is discontinuous. However, the differences between the measured and predicted volumetric strains varied from less than $0.005 \epsilon_{v}$ in tests which were prepared at relatively high degrees of saturation (eg. tests $18 \mathrm{~F} 89 \mathrm{c} 037$ and $18 \mathrm{~F} 78 \mathrm{c} 030$ ), to $0.02 \epsilon_{v}$ in tests with similar and slightly lower degrees of saturation (eg. tests $18 \mathrm{~F} 77 \mathrm{c} 083$ and 18F76c075). Variations of $0.02 \epsilon_{v}$ between the measured and actual volumetric strains is significant, as these sample experienced total volumetric strains of 3 to $3.5 \%$ Some tests, for example, test $18 \mathrm{~F} 74 \mathrm{c} 048$, were prepared at lower degrees of saturation but had a moderate error of approximately $0.008 \epsilon_{v}$ between the actual and predicted volumetric strains. This suggests that the variation between the measured volume changes and the volume changes predicted from using Boyle's Law is not related to the degree of saturation within the range of degrees of saturation tested in this study. The magnitude of the CSR also did not appear to have a consistent effect on the difference between the actual and predicted volume changes. For example, test $18 \mathrm{~F} 86 \mathrm{c} 030$ had a large error of $0.020 \epsilon_{v}$ between the measured and predicted volumetric strains while test $18 \mathrm{~F} 78 \mathrm{c} 030$, which was tested at the same CSR and at a similar void ratio, had a smaller error of $0.003 \epsilon_{v}$ between the measured and predicted volumetric strains. From Fig. 8, it appears that Boyle's Law predicted the volume changes in denser samples better than in looser samples, however, there were other tests, which were prepared at slightly higher void ratios, where Boyle's Law predicted the volumetric strains within $0.005 \epsilon_{v}$ of the measured volumetric strains as shown in Fig. 11.

Fig. 12 shows the number of tests with different magnitudes of error between the predicted and actual volume changes that resulted in the samples tested in this study. Most of the tests had a variation of less than a $0.005 \epsilon_{v}$ between the measured and predicted volumetric strains, however, there were a number of tests which had larger differences between the predicted and measured volumetric strains. Boyle's Law does not account for the volume of air that dissolves into the water, a time dependent process which would cause a further decrease in the measured sample's volume. According to Henry's Law, the maximum amount of dissolved air per kilogram of water at atmospheric pressure and at $25^{\circ} \mathrm{C}$ is approximately $0.023 \mathrm{~g} / \mathrm{kg}$ and under the same conditions, the density of air is approximately $1.19 \mathrm{~kg} / \mathrm{m}^{3}$. Therefore, up to $19 \mathrm{~mm}^{3}$ of air can be dissolved per $1,000 \mathrm{~mm}^{3}$ of water. The volume of water in the unsaturated samples during the cyclic loading stage varied from 250,000 to $300,000 \mathrm{~mm}^{3}$ depending on the degree of saturation and therefore, the volume of dissolved air can vary from approximately 4,750 to $5,700 \mathrm{~mm}^{3}$ and this corresponds to less than $0.005 \epsilon_{v}$ variation in the predicted volumetric strains. A correction allowing for the volume of air that dissolves in the samples was not ap- 
plied in this study as the actual amounts of dissolved air within each of the samples were not determined. However, as dissolved air can cause variations of up to an extra $0.005 \epsilon_{v}$ in the predicted volume strains, this suggests that Boyle's Law also predicted plausible volumetric strains in the 7 samples that varied by 0.005 to $0.01 \epsilon_{v}$ from the measured volumetric strains. Errors in the predicted volume changes of the samples could also be caused by blockages in the air line that was connected to the top of the samples. In several tests, water was observed to flow out of the sample into the air line and the air pressure transducer. These blockages caused unreliable air pressure measurements and incorrect volume predictions. However, it was clear when this occurred as jumps in the air pressures or constantly low air pressures were observed.

Larger variations in volumetric strain, $>0.01 \epsilon_{v}$, occurred in 8 of the 28 samples tested in this study and the source of error in these tests is not known. It is unlikely that the air pressures measured in these tests were incorrect as unrealistic corrections of up to $40 \mathrm{kPa}$ are required for the predicted volume changes to be consistent with the measured volume changes of the sample. Boyle's Law consistently under predicted the amount that samples compressed, which suggests that there is a systematic error in either using the cell water to measure the volume changes of the sample, or in using Boyle's Law to estimate the volumetric strains in the unsaturated samples. It is possible that there were air bubbles in the triaxial cell that compressed and dissolved during these tests, resulting in an over estimation of the measured decrease in the volume of the samples. However, the initial sizes of air bubbles that may have been present in the cell during the tests were not measured. Relatively large samples were used in this study and as a result, the differences between the predicted and the measured volumetric strains were small, up to $2 \%$, relative to the sample's total volume, which suggests that and the resulting errors in the calculated degree of saturation and effective stresses within the samples were also small. However, the samples also experienced compressive volumetric strains of typically between 2 to $6 \%$. Therefore, a difference of more than $0.01 \epsilon_{v}$ between the measured and predicted volumetric strains in the samples from using Boyle's Law, is significant. However, the majority of the samples had less than $0.005 \epsilon_{v}$ difference between the measured and predicted volumetric strains. Further volumetric strains of up to $0.005 \epsilon_{v}$ could be applied as an extra volume correction on the predicted volumetric strains to account for the dissolution of air, which would reduce the difference between the measured and predicted volumetric strains. However, further work is required to more accurately determine the amount of dissolved air within the samples.

\section{Conclusion}

The effects of the degree of saturation, CSR and density on the pore air and water pressure responses, and on the volumetric response of unsaturated samples, have been investigated. More rapid increases in the pore pressures were observed in looser samples that were tested at higher degrees of saturation and CSRs. Boyle's Law was used to relate the increases in pore air pressures with the compressive volumetric strains, within less than $0.001 \epsilon_{v}$ of the measured volumetric strains for the majority of samples. The corresponding variation in volume was relatively small compared to the large volumes of the samples tested in this study, but was significant when compared to the volume changes that samples experienced during the cyclic loading stages. The variation between the measured and predicted volumetric strains can be reduced by up to $0.005 \epsilon_{v}$ if the dissolution of air is considered, however, further study is required to determine the actual volumes of air that dissolves within the samples. Further study is also required to determine whether Boyle's Law can reliably predict the volume or air pressure changes within smaller samples, typically tested in unsaturated cyclic liquefaction studies, that are more sensitive to errors in volume measurements.

\section{References}

[1] Bishop, A.W., Blight, G.E., Geotechnique, 13, 177197 (1963)

[2] Unno, T., Kazama, M., Uzuoka, R., Sento, N., Soils \& Found. 48, 87-99 (2008)

[3] Uzuoka, R., Unno, T., Sento, N., Kazama, M., Unsat. Soils: Research \& Applications, 783-789 (2014)

[4] Okamura, M., Soga, Y., Soils \& Found., 46, 695-700 (2006)

[5] Wang, H., Koseki, J., Sato, T., Chiaro, G., Tian, J.T., Soils \& Found., 56, 732-744 (2016)

[6] Hu, D., Lo, S.R., OttawaGeo, 1108-1114 (2007)

[7] Macari, E. J., Parker, J. K., Costes, N. C. Geotech. Test. J., 20, 103-109 (1997)

[8] Gachet, P., Geiser, F., Laloui, L., Vulliet, L. Geotech. Test. J., 30, 98-103 (2006)

[9] Kimoto, S., Oka, F., Fukutani, J., Yabuki, T., Nakashima, K., Soils \& Found., 51, 663-681 (2011)

[10] Tsukamoto, Y., Kawabe, S., Matsumoto, J., Hagiwara, S., Soils \& Found., 54, 1094-1103 (2014)

[11] Technical Working Group (TWG), TWG, Reference tests, TWG Report, (2013)

[12] Fredlund, D.G., Rahardjo, H., Fredlund, M.D. Unsaturated Soil Mechanics in Engineering Practice (John Wiley \& Sons Inc., 2012)

[13] Ng, C.W.W., Menzies, B., Advanced Unsaturated Soil Mechanics and Engineering (CRC Press, 2017) 